\title{
Prevalencia de los trastornos de la voz en los docentes de la Universidad del Magdalena, Colombia, 2017-2018
}

\section{Prevalence of voice disorders in teachers Universidad del Magdalena, Colombia 2017-2018}

\author{
Francisco Revollo-Zúñiga ${ }^{1}$ (D) , Javier Hernández-Blanco² (D), Alexander Salazar-Ceballos ${ }^{3}$ (D), Andrea Dávila-Cueto ${ }^{4}$ \\ 1. Universidad del Magdalena. Santa Marta, Colombia. Correo: franciscorevolloz@gmail.com - https://orcid.org/0000-0002-7713-0263 \\ 2. Universidad del Magdalena. Santa Marta, Colombia. Correo: jhernandezblanco@gmail.com - https://orcid.org/0000-0003-0473-8814 \\ 3. Universidad del Magdalena. Santa Marta, Colombia. Correo: asalazar@unimagdalena.edu.co - https://orcid.org/0000-0002-0708-87924 \\ 4. Universidad del Magdalena. Santa Marta, Colombia. Correo: andreadavilacueto@gmail.com - https://orcid.org/0000-0002-7970-5497 \\ Tipología: Artículo de investigación científica y tecnológica \\ Para citar este artículo: Revollo-Zúñiga F, Hernández-Blanco J, Salazar-Ceballos A, Dávila-Cueto A. Prevalencia de los trastornos de la voz en los docentes de la Universidad del \\ Magdalena, Colombia, 2017-2018. Duazary. 2020 abril - junio; 17(2): 1 - 9. Doi: http://dx.doi.org/10.21676/2389783X.3235 \\ Recibido en febrero 06 de 2019 \\ Aceptado en septiembre 04 de 2019 \\ Publicado en línea en diciembre 02 de 2019
}

Palabras

clave: voz; docentes; factores de riesgo.

Keywords: Voice; Teachers; Risk factors.

\section{RESUMEN}

Se realizó un estudio descriptivo de corte transversal para determinar la prevalencia de los trastornos de la voz y los factores de riesgo asociados en los docentes de la Universidad del Magdalena (Colombia) en el periodo 2017-2018. Se incluyeron 263 docentes, se recolectaron datos de sexo, edad, estrato, programa académico, años de trabajo, horas de clase, alumnos por salón, uso de amplificación, toma de licor, tabaquismo y diagnóstico de reflujo gastroesofágico y se les aplico el índice de incapacidad vocal 10 (VHI-10). Los docentes con puntaje mayor o igual a 11 fueron valorados en consulta bajo nasofibrolaringoscopia. Se observó que el $20 \%$ de los docentes presentó incapacidad vocal, con un puntaje en VHI-10 mayor o igual a 11. En los análisis hubo asociación estadísticamente significativa entre incapacidad vocal y el diagnóstico médico de reflujo. Al evaluar a 28 profesores con nasofibrolaringoscopia, se determinó que cerca del 54\% (15/28) presentaron actividad supraglótica y fonación con bandas. Los docentes son un grupo de personas con alteraciones de la voz mayores que la población general y requieren un entrenamiento especial y específico en su conservación. Independiente de las condiciones particulares de la actividad de enseñanza, la enfermedad por reflujo gastroesofágico mostró estar asociada a incapacidad vocal.

\section{ABSTRACT}

A cross-sectional descriptive study was carried out to determine the prevalence of voice disorders and associated risk factors in teachers at the Universidad del Magdalena (Colombia) 2017-2018. 263 teachers were included, and data on sex, age, socioeconomic stratum, academic program, years of teaching work, number of class hours, number of students per classroom, use of amplification, frequency of liquor intake, smoking, medical diagnosis of gastroesophageal reflux, and the Voice Disability Index 10 (VHI 10) were collected. Teachers with scores greater than or equal to 11 were valued in consultation with Nasofibrolaringoscopy. It was observed that $20 \%$ of teachers presented a vocal disability with a score of VHI 10 greater than or equal to 11 . In the analyses there was statistically significant association between vocal disability and medical reflux diagnosis. We evaluated 28 professors with Nasofibrolaringoscopy, it was determined that about 54\% (15/28) presented supraglottic activity and phonation with vocal false cords. Teachers are a group of people with an index of alterations of the voice greater than the general population and require special training and specific in their conservation. Independent of the particular conditions of the teaching activity, gastroesophageal reflux disease showed to be associated with vocal incapacity. 


\section{INTRODUCCIÓN}

Los docentes pertenecen a un grupo poblacional que de forma rutinaria dispone de la voz para poder laborar. La afectación transitoria o permanente de esta necesita de atención, ya que el deterioro en la calidad vocal de un profesor interfiere con la realización de sus funciones, llegando hasta comprometer su bienestar psicológico y social. Los profesores con síntomas de afectación laríngea exceden en frecuencia a la población general, lo que reafirma que los individuos consagrados a esta labor se encuentran en un riesgo mayor y precisan ser formados en cómo modular la demanda vocal de su trabajo. Los profesores tienen la función única dentro del seno de la sociedad de ser, junto con los padres de familia, los formadores de los niños y jóvenes de un país. Toda ausencia laboral de un docente perjudica no solo al profesor, sino a los estudiantes y a la empresa educativa.

Entre los puntos de interés y las variables que merecen tenerse en cuenta para explicar la alta frecuencia de patología vocal en los docentes podemos encontrar: horas de docencia día, tipo de asignatura, estudiantes por aula, el uso o no de amplificación, años de labor, el tipo de formación que se instruye (preescolar, primaria, bachillerato, universidad), hábitos tóxicos (licor, cigarrillo), entre otros.

En las conclusiones de los estudios sobre el tema se evidencia que los docentes son más propensos a presentar alteraciones del aparato fonatorio, $y$ existe una tasa de enfermedad laboral en los profesores entre el $20 \%$ y el $57 \%^{1,2}$. En España, por ejemplo, se alcanza una prevalencia del $57 \%$ de alteraciones vocales, y en países vecinos, como Brasil y Argentina, esta es de $18,6 \%$ y $33,3 \%$, respectivamente ${ }^{3-5}$.

En nuestro país se han llevado a cabo pocas investigaciones en el gremio de educadores que busquen la etiología de los padecimientos vocales. Estos estudios se destacan por su enfoque en las condiciones laborales y ambientales del docente; sin embargo, es importante determinar qué predispone a los docentes a desarrollar un trastorno vocal y cuáles son las lesiones laríngeas que presentan los profesores afectados de la voz, con miras a realizarles el manejo precoz adecuado.

Para valorar la alteración vocal encontramos diferentes pruebas. Entre ellas, el VHI fue desarrollado con el fin de cuantificar el impacto percibido por un sujeto afectado por un trastorno vocal. EI VHI es el cuestionario más versátil y fácil de completar por el paciente y contiene la información más relevante acerca de la calidad de vida relacionada con la voz. Este instrumento contiene 30 ítems, si bien existe la versión acortada de 10 ítems que tiene la misma validez y fiabilidad. En la versión acortada (VHI-10), las preguntas 1 a 5 reflejan el aspecto funcional; las preguntas 6,7 y 10 , el aspecto físico, y las preguntas 8 y 9 , el rubro emocional ${ }^{6}$.

Teniendo en cuenta la magnitud de la patología vocal en los maestros, este estudio tiene por objeto determinar la prevalencia de los trastornos de la voz y los factores de riesgo asociados en los docentes de la Universidad del Magdalena.

\section{MATERIALES Y MÉTODOS}

\section{Tipo de investigación}

Estudio descriptivo, de corte transversal.

\section{Población}

La Universidad del Magdalena en el momento del estudio contaba con 991 docentes.

\section{Instrumentos}

A todos los docentes entre el 1 de julio y el 1 de diciembre 2017 se les envió por correo electrónico una encuesta creada en Google formularios donde se les pedía de forma voluntaria, y previa autorización por consentimiento informado, los datos de sexo, edad, estrato socioeconómico, programa académico al que pertenecían, años de trabajo docente, número de horas de clase, número de alumnos por salón de clase, uso de amplificación, frecuencia de toma de licor, tabaquismo y diagnóstico médico de reflujo gastroesofágico. En esta encuesta estaba inserto el índice de incapacidad vocal 10 (Voice Handicap Index VHI-10). EI VHI-10 clasifica la incapacidad vocal en leve (10 puntos o menos), moderada (11 a 20 puntos), severa 
(21 a 30 puntos) y grave (31 a 40 puntos). Los pacientes con puntaje igual o superior a 11 entraron a la segunda fase del estudio, donde eran valorados en consulta por un mismo médico otorrinolaringólogo con nasofibrolaringoscopia (Olympus ENF-P4).

\section{Análisis estadístico}

Se utilizó el programa para ciencias sociales SPSS versión 20 (IBM, EE. UU.) para el procesamiento de datos.

\section{Declaración sobre aspectos éticos}

Este estudio adoptó las normas establecidas en la Declaración de Helsinki de 1975 y en la Resolución 8430 de 1993 del Ministerio de Salud de Colombia para la investigación con seres humanos. Se presentó el consentimiento informado y se protegió el bienestar e integridad de los participantes a través de la confidencialidad y el derecho a la no participación y el retiro y la devolución de resultados.

\section{RESULTADOS}

El grupo de estudio quedó conformado por 263 individuos, quienes fueron los que respondieron la encuesta y cumplían los criterios de inclusión:

- Ser docente de la Universidad del Magdalena.

- Tener por lo menos un año continuo de ejercicio educativo.

- Tener como actividad principal dictar clases.

- Dar su autorización para participar en el estudio.

En la Tabla 1 se presenta la caracterización de la población de docentes universitarios. Se observa que casi el $56 \%$ eran hombres, cerca del $66 \%$ toma licor ocasionalmente, casi el $95 \%$ no fuma, el $80 \%$ reside entre los estratos 3 y 4 , y solo el $3 \%$ utiliza algún tipo de amplificación durante las clases. Las facultades de salud e ingenierías fueron en las que más docentes respondieron la encuesta, entre el $27 \%$ y el $29 \%$ respectivamente.

En relación con la frecuencia de casos de incapacidad vocal de los docentes universitarios que respondieron la encuesta, se observó que cerca del $17 \%$ presentó incapacidad vocal moderada, y cerca del 3\% presentó incapacidad vocal severa; ninguno presentó incapacidad vocal grave (Tabla 2).

Se procedió a dicotomizar la variable de los niveles de incapacidad vocal tomando, por un lado, la incapacidad leve y uniendo, por otro lado, los niveles de incapacidad moderada y severa. El nivel de incapacidad leve estuvo representado en casi el $80 \%$ de la población $(79,85 \%)$. Sucesivamente, para llevar a cabo los análisis por chi cuadrado, se dicotomizaron las demás variables. En los análisis por chi cuadrado solo se observó asociación estadísticamente significativa entre incapacidad vocal y el diagnóstico médico de reflujo $\left(X^{2}=12,45\right.$ y $p=0,00041$ ) (Tabla 3).

En la Tabla 4 se presentan los análisis por chi cuadrado entre el índice de incapacidad vocal (leve vs. moderada-severa) y el número de estudiantes y el número de horas de clase por semana, donde no se observó ninguna asociación estadísticamente significativa.

En la Tabla 5 se observan las frecuencias de los profesores universitarios que presentaron incapacidad vocal y que fueron evaluados clínicamente. Solo se evaluaron 28 profesores de 53 que presentaron incapacidad moderada y severa porque estos fueron los que acudieron voluntariamente a la cita con el especialista en otorrinolaringología para la realización de nasofibrolaringoscopia. Según la tabla, cerca del $54 \%$ (15/28) de los profesores presentaron actividad supraglótica y fonación con bandas. Así mismo se detectaron dos profesores con una lesión supraglótica a nivel de cuerdas falsas, quienes se canalizaron para microcirugía laríngea, la cual estaba pendiente al cierre de este estudio.

En la Tabla 6 se observa que no existe relación entre diagnóstico médico de reflujo y los hallazgos de la nasofibrolaringoscopia. Esta tabla se presenta a manera de ejercicio, pues estadísticamente no se acepta el análisis por chi cuadrado dado que varias casillas tuvieron valores menores de 5 .

No se encontró asociación entre la incapacidad vocal y los grupos de edad, ni entre la incapacidad vocal y diferentes agrupaciones por número de estudiantes, ni por el uso de aparatos de amplificación (datos no mostrados). 
En Tabla 7 se muestran las diez preguntas resueltas por los participantes del índice de incapacidad vocal adaptado al idioma español.

Tabla 1. Caracterización de la población de docentes universitarios.

\begin{tabular}{|c|c|c|c|}
\hline Variables & Categoría & Frecuencia & $\%$ \\
\hline \multirow{2}{*}{ Sexo } & Mujer & 116 & 44,11 \\
\hline & Hombre & 147 & 55,89 \\
\hline \multirow{4}{*}{ Edad } & Menores de 40 & 85 & 32,57 \\
\hline & Menores de 50 & 81 & 31,03 \\
\hline & Mayores de 50 & 77 & 29,50 \\
\hline & Total & 261 & 100 \\
\hline \multirow{4}{*}{ ¿Con qué frecuencia toma licor? } & Nunca & 78 & 29,89 \\
\hline & Ocasionalmente & 171 & 65,52 \\
\hline & Semanalmente & 12 & 4,60 \\
\hline & Total & 261 & 100 \\
\hline \multirow[t]{3}{*}{ ¿Usted fuma? } & No & 246 & 94,62 \\
\hline & Sí & 14 & 5,38 \\
\hline & Total & 260 & 100 \\
\hline \multirow[t]{7}{*}{$\begin{array}{l}\text { Estrato socioeconómico donde reside } \\
\text { actualmente }\end{array}$} & 1 & 5 & 1,92 \\
\hline & 2 & 20 & 7,66 \\
\hline & 3 & 102 & 39,08 \\
\hline & 4 & 81 & 31,03 \\
\hline & 5 & 27 & 10,34 \\
\hline & 6 & 26 & 9,96 \\
\hline & Total & 261 & 100 \\
\hline \multirow[t]{3}{*}{$\begin{array}{l}\text { En la mayoría de sus clases utiliza algún tipo } \\
\text { de amplificación }\end{array}$} & No & 252 & 96,55 \\
\hline & Sí & 9 & 3,45 \\
\hline & Total & 261 & 100 \\
\hline \multirow[t]{8}{*}{ Facultad } & Básicas & 14 & 5,32 \\
\hline & Educación & 20 & 7,60 \\
\hline & Empresas & 45 & 17,11 \\
\hline & Humanidades & 28 & 10,65 \\
\hline & Idiomas-generales & 9 & 3,42 \\
\hline & Ingeniería & 76 & 28,90 \\
\hline & Salud & 71 & 27 \\
\hline & Total & 263 & 100 \\
\hline
\end{tabular}

Tabla 2. Distribución de frecuencias de índice de incapacidad vocal.

\begin{tabular}{|c|c|c|}
\hline Incapacidad vocal & Frecuencia & Porcentaje \\
\hline Leve & 210 & $79,85 \%$ \\
\hline Moderada & 46 & $17,49 \%$ \\
\hline Severa & 7 & $2,66 \%$ \\
\hline Total & 263 & $100,00 \%$ \\
\hline
\end{tabular}


Francisco Revollo-Zúñiga, Javier Hernández-Blanco, Alexander Salazar-Ceballos, Andrea Dávila-Cueto

Tabla 3. Incapacidad vocal (leve vs. moderada-severa) relacionada con diferentes variables.

\begin{tabular}{|c|c|c|c|c|c|c|}
\hline Variables & Categorías & Leve & Moderada-severa & n (\%) & Chi cuadrado & Valor $p$ \\
\hline \multirow[t]{2}{*}{ Sexo } & Femenino & 91 & 25 & $116(44,11)$ & 0,25 & $p>0,05$ \\
\hline & Masculino & 119 & 28 & $121(55,89)$ & & \\
\hline \multirow[t]{2}{*}{ Estrato } & 1,2 y 3 & 100 & 27 & $127(48,85)$ & 0,11 & $p>0,05$ \\
\hline & 4,5, y 6 & 107 & 26 & $133(51,15)$ & & \\
\hline \multirow[t]{2}{*}{$\begin{array}{l}\text { Diagnóstico } \\
\text { médico de } \\
\text { reflujo }\end{array}$} & No & 182 & 36 & $218(83,85)$ & 12,45 & $\mathrm{p}<0,05$ \\
\hline & $\mathrm{Si}$ & 25 & 17 & $42(16,15)$ & & \\
\hline \multirow[t]{2}{*}{ Fuma } & No & 195 & 51 & $246(94,62)$ & 0,33 & $p>0,05$ \\
\hline & $\mathrm{Si}$ & 12 & 2 & $14(5,38)$ & & \\
\hline \multirow[t]{3}{*}{$\begin{array}{c}\text { Años de } \\
\text { trabajo } \\
\text { docente }\end{array}$} & $\begin{array}{c}\text { Menor o igual a } 5 \\
\text { años }\end{array}$ & 57 & 15 & $72(27)$ & 0,46 & $p>0,05$ \\
\hline & $\begin{array}{c}\text { Más de } 5 \text { años y } \\
\text { menor o igual a } 10 \\
\text { años }\end{array}$ & 49 & 10 & $59(22,7)$ & & \\
\hline & Más de 10 años & 101 & 27 & $128(49,42)$ & & \\
\hline \multirow[t]{3}{*}{$\begin{array}{l}\text { Frecuencia de } \\
\text { toma de licor }\end{array}$} & Nunca & 62 & 16 & $78(29,88)$ & 1,36 & $p>0,05$ \\
\hline & Ocasional & 138 & 33 & $171(65,57)$ & & \\
\hline & Semanal & 8 & 4 & $12(4,59)$ & & \\
\hline
\end{tabular}

Tabla 4. Índice de incapacidad vocal (leve vs. moderada-severa) relacionada con número de estudiantes y número de horas semanales.

\begin{tabular}{|c|c|c|c|c|c|c|}
\hline Variables & Categorías & Leve & $\begin{array}{c}\text { Moderada- } \\
\text { severa }\end{array}$ & $\begin{array}{c}\% \\
\text { por fila }\end{array}$ & $\begin{array}{c}\text { Chi } \\
\text { cuadrado }\end{array}$ & Valor p \\
\hline $\begin{array}{c}\text { Número de } \\
\text { estudiantes }\end{array}$ & $\begin{array}{c}\text { Menor o igual a } \\
20\end{array}$ & 46 & 6 & 19,5 & 3,153 & $p>0,05$ \\
\hline & $\begin{array}{c}\text { Más de 20 y } \\
\text { menor o igual a } \\
40\end{array}$ & 139 & 41 & 70,3 & & \\
\hline & Más de 40 & 19 & 5 & 9,3 & & \\
\hline $\begin{array}{c}\text { Número de } \\
\text { horas semana }\end{array}$ & Menos de 20 & 152 & 42 & 76,08 & 0,7898 & $p>05$ \\
\hline & Más o igual a 20 & 51 & 10 & 23,92 & & \\
\hline & \% por columna & 79,6 & 20,39 & & & \\
\hline
\end{tabular}


Tabla 5. Relación entre profesores universitarios que presentaron incapacidad vocal y que fueron evaluados clínicamente.

\begin{tabular}{|c|c|c|c|c|}
\hline Nasofibro & Moderada & Severa & Total & $\%$ \\
\hline 1 Actividad supraglótica fonación con bandas & 12 & 3 & 15 & 53,57 \\
\hline 2 Laringitis RGE & 9 & 1 & 10 & 35,71 \\
\hline 3 Normal & 1 & 0 & 1 & 3,57 \\
\hline 4 Masa laríngea & 2 & 0 & 2 & 7,14 \\
\hline Total & 24 & 4 & 28 & 100 \\
\hline
\end{tabular}

Tabla 6. Relación entre diagnóstico médico de reflujo y nasofibrolaringoscopia.

\begin{tabular}{|c|c|c|c|c|c|}
\hline Nasofibrolaringoscopia & No & Si & Total & Chi cuadrado & Valor $\mathbf{p}$ \\
\hline $\begin{array}{c}\text { Actividad supraglótica fonación con } \\
\text { bandas }\end{array}$ & 12 & 3 & 15 & 3,24 & $p>0,05$ \\
\hline Laringitis RGE & 5 & 5 & 10 & & \\
\hline Normal & 1 & 0 & 1 & & \\
\hline Masa laríngea & 1 & 1 & 2 & & \\
\hline Total & 19 & 9 & 28 & & \\
\hline
\end{tabular}

Tabla 7. Índice de incapacidad vocal acortado (VHI-10), adaptación al español.

\begin{tabular}{|c|c|c|c|c|c|}
\hline & Nunca & Casi nunca & A veces & Casi Siempre & Siempre \\
\hline $\begin{array}{c}\text { F1. La gente me oye con dificultad debido } \\
\text { a mi voz }\end{array}$ & 0 & 1 & 2 & 3 & 4 \\
\hline $\begin{array}{c}\text { F2. La gente no me entiende en sitios } \\
\text { ruidosos }\end{array}$ & 0 & 1 & 2 & 3 & 4 \\
\hline $\begin{array}{c}\text { F8. Mis problemas con la voz alteran mi } \\
\text { vida personal y social }\end{array}$ & 0 & 1 & 2 & 3 & 4 \\
\hline $\begin{array}{c}\text { F9. Me siento desplazado de las } \\
\text { conversaciones por mi voz }\end{array}$ & 0 & 1 & 2 & 3 & 4 \\
\hline $\begin{array}{c}\text { F10. Mi problema con la voz afecta mi } \\
\text { rendimiento laboral }\end{array}$ & 0 & 1 & 2 & 3 & 4 \\
\hline $\begin{array}{c}\text { P5. Siento que necesito forzar la garganta } \\
\text { para producir la voz }\end{array}$ & 0 & 1 & 2 & 3 & 4 \\
\hline $\begin{array}{c}\text { P6. La calidad de mi voz es impredecible } \\
\text { E4. Mi voz me molesta }\end{array}$ & 0 & 1 & 2 & 3 & 4 \\
\hline $\begin{array}{c}\text { E6. Mi voz me hace sentir incapacitado } \\
\text { con la voz?” }\end{array}$ & 0 & 1 & 2 & 3 & 4 \\
\hline P3. La gente me pregunta “ique te pasa & 0 & 1 & 2 & 3 & 4 \\
\hline
\end{tabular}




\section{DISCUSIÓN}

Se documenta estadísticamente que la participación de trabajadores que dependen de la voz para desarrollar su actividad laboral puede alcanzar hasta un tercio de la población trabajadora de un país. En esta situación encontramos a docentes, locutores, trabajadores sociales, operadores telefónicos y todo aquel personal que dentro de sus funciones establece la atención al publico ${ }^{7-9}$.

La relación cercana que tienen los docentes con su voz como herramienta de trabajo permite clasificarlos como un grupo de personas con un índice de alteraciones de la voz mayor que la población general. En este trabajo la prevalencia de trastornos de la voz fue de $20,15 \%$, puntaje mayor o igual a 11 en el índice de incapacidad vocal acortado (VHI-10), datos que se contrastan con estudios anteriores de autores como Agostini y Pérez, donde muestran docentes en Perú con prevalencia del $44 \%$, en Brasil con 18,6\%, en Argentina con $33,3 \%$ y en España con $57 \%^{10-12}$.

La investigación permitió demostrar disfonías funcionales (actividad supraglótica y fonación con bandas) en el 53,57\% de los docentes con un índice de incapacidad moderada y severa, a los que se les realizó nasofibrolaringoscopia. No se encontró asociación estadísticamente significativa entre incapacidad vocal, número de estudiantes, edad, sexo y número de horas de clase por semana. Esta información recolectada se aparta de estudios donde se expone que en los docentes jóvenes con menos de 10 años de ejercicio predominan cuadros patológicos por abuso y mal uso de la voz.

La gran limitante del estudio fue que solo se evaluaron bajo visión endoscópica 28 profesores de 53 que presentaron incapacidad moderada y severa, porque estos fueron los únicos que decidieron continuar con el estudio endoscópico de la laringe ${ }^{13-}$ ${ }^{16}$. En este punto vale la pena precisar que existen dispositivos para diagnósticos endoscópicos más precisos, como la laringoestroboscopia, de los cuales carece la ciudad origen del estudio y que serían motivo de otra investigación.

En el caso de los educadores, el uso incorrecto en la técnica vocal y el diagnóstico previo de enfermedad por reflujo gastroesofágico originan problemas en su bienestar físico, psicológico y social, influyendo tanto en la salud como en su calidad de vida y afectando el desempeño correcto de su profesión. La presencia de enfermedad por reflujo gastroesofágico puede requerir de una prueba adicional de $\mathrm{pH}$-metría para buscar una asociación más fuerte en cuanto a la fisiopatología precisa de su relación con la disfonía.

Desafortunadamente, en nuestro país no se da relevancia al uso apropiado de la voz y entre los docentes se desconocen los cuidados para conservarla. El eje central de la prevención es desarrollar conciencia respecto a la importancia del uso de la voz como instrumento de trabajo. Las acciones informativas como volantes o recomendaciones teóricas del cuidado de la voz no funcionan si no se acompañan de acciones prácticas educativas y formativas hacia los docentes ${ }^{17-20}$.

\section{CONCLUSIÓN}

1. Teniendo en cuenta el índice de incapacidad vocal acortado ( $\mathrm{VHI}-10)$, existe una prevalencia del $20,15 \%$ de disfonía en profesores de la Universidad del Magdalena.

2. Los docentes disfónicos encontrados presentan una alteración moderada a severa en el VHI-10.

3. La condición de profesor es un factor de riesgo para trastornos de la voz.

4. No se demostró relación entre alteración de la voz, número de estudiantes, edad, sexo y número de horas de clase por semana.

5. Se documentaron disfonías funcionales (actividad supraglótica y fonación con bandas) en el $53,57 \%$ de los docentes con un índice de incapacidad moderada y severa, a los que se les realizó nasofibrolaringoscopia.

6. Se refuerza la relación existente entre enfermedad por reflujo gastroesofágico y alteración vocal.

7. Existe completo desconocimiento de las medidas para obtener una técnica vocal adecuada en la población profesoral.

8. Se requieren acciones prácticas educativas y formativas hacia el cuidado de la voz de la población docente. 


\section{AGRADECIMIENTOS}

Los autores desean expresar que se contó en todo momento con la colaboración de la planta docente y de la Vicerrectoría de Investigación de la Universidad del Magdalena. Sin ellos no hubiera sido posible llevar a cabo este estudio. Se menciona también el aporte realizado por la estudiante de medicina Johana Parra.

\section{DECLARACIÓN SOBRE CONFLICTOS DE INTERÉS}

Los autores declaran no tener conflictos de interés.

\section{CONTRIBUCION DE LOS AUTORES}

Revollo-Zúñiga F: idea de investigación, desarrollo del proyecto, realización de estudios endoscópicos, elaboración del manuscrito.

Hernández-Blanco J, Salazar-Ceballos A: diseño metodológico, análisis estadístico e interpretación de datos.

Dávila-Cueto A: recolección de datos y elaboración de la base de datos.

Este trabajo recibió financiación de la Convocatoria Interna Capital Semilla Universidad del Magdalena (acta de inicio 13 de marzo del 2017).

\section{REFERENCIAS BIBLIOGRÁFICAS}

1. Cantor Cutiva LC, Vogel I, Burdorf A. Voice disorders in teachers and their associations with work-related factors: a systematic review. J Commun Dis. 2013; 46: 143-155. Doi: https://doi.org/10.1016/j.jcomdis.2013.01.001.

2. Unesco. Condiciones de Trabajo y Salud Docente [internet]. Unesco, Oficina Regional de Educación para América Latina y el Caribe, Argentina, Chile, Ecuador, México, Perú, Uruguay y Chile. 2005. Disponible en: https://connect.unesco.org/danana/auth/url_default/welcome.cgi.

3. Varela A. Prevalencia de los trastornos de la voz y las variables asociadas a los mismos en docentes de dos colegios de Bogotá [internet] [trabajo de grado Especialista en Salud Ocupacional]. Bogotá: Pontificia Universidad
Javeriana. Facultad de Enfermería; 2009. Disponible en: https://www.javeriana.edu.co/biblos/tesis/enf ermeria/tesis75.pdf.

4. Preciado L, Pérez FC. Epidemiological study of voice disorders among teaching professionals of La Rioja, Spain. J Voice. 2008; 22: 489-508.

Doi: https://doi.org/10.1016/j.jvoice.2006. 11.008.

5. Puyuelo $M$, Llinás $M$. Problemas de voz en docentes. Rev. Logop Fon Audiol. 1992: 12(2): 76-84. Doi: https://doi.org/10.1016/S02144603(92)75536-3.

6. Núñez-Batalla $F$, Corte-Santos $P$, SeñarisGonzález B, Llorente-Pendás JL, Górriz-Gil C, Suárez-Nieto $C$. Adaptation and validation to the Spanish of the Voice Handicap Index (VHI-30) and its shortened version (VHI-10). Acta Otorrinolaringol Esp 2007; 58(9): 386-392. Doi: https://doi.org/10.1016/S2173-5735(07)703769.

7. Roy N, Merrill RM, Thibeault S, Parsa RA, Gray SD, Smith EM. Prevalence of voice disorders in teachers and the general population. J Speech Lang Hear Res. 2004; 47: 281-293. Doi: https://doi.org/10.1044/1092-4388(2004/023).

8. Vilkman E. Occupational safety health aspects of voice and speech professions. Folia Phoniatr Logop 2004; 56(4): 220-253. Doi: https://doi.org/10.1159/000078344.

9. Behlau M, Zambon F, Guerrieri AC, Roy N. Epidemiology of voice disorders in Teachers and Nonteachers in Brazil: Prevalence and adverse effects. J Voice. 2012; 26(5): 665-75. Doi: https://doi.org/10.1016/j.jvoice.2011.09.0 10.

10. Agostini-Zampieron M, Barlatey-Frontera C, Barlatey- Frontera MF, Arca-Fabre A. Prevalencia de disfonías funcionales en docentes argentinos. Aten Fam. 2013; 20(3): 8185. Doi: https://doi.org/10.1016/S14058871(16)30097-9.

11. Pérez C. Preciado J. Calzada M. Examen de la función vocal y análisis acústico de 905 docentes 
de La Rioja. Acta Otorrinolaringol Esp. 2005;

56(6): 261-72. Doi: https://doi.org/10.1016/S0001-6519(05)78612$\mathrm{X}$.

12. Van Houtte E, Claeys S, Wuyts F, Van Lierde K. The impact of voice disorders among teachers: Vocal complaints, treatment-seeking behavior, knowledge of vocal care, and voice-related absenteeism. J Voice 2011; 25(5): 570-575. Doi: https://doi.org/10.1016/j.jvoice.2010.04.0 08.

13. Korn GP, Park SW, De Lima Pontes AA, Pontes P. Vocal symptoms and associated risk factors between male and female university teachers. Int Arch Otorhinolaryngol 2018; 22(3): 271-279. Doi: https://doi.org/10.1055/s-0037-1606604.

14. Rodrigues G, Zambon F, Mathieson L, Behlau M. Vocal tract discomfort in teachers: Its relationship to self-reported voice disorders. J Voice 2013; 27(4): 473-480. Doi: https://doi.org/10.1016/j.jvoice.2013.01.0 05.

15. Cantor-Cutiva LC, Burdorf A. Medical Costs and Productivity Costs Related to Voice Symptoms in Colombian Teachers. J Voice. 2015; 29(6): 77684.

Doi: https://doi.org/10.1016/j.jvoice.2015.01.0 05.
16. Hartley NA, Thibeault SL. Systemic Hydration: Relating Science to Clinical Practice in Vocal Health. J Voice. 2014; 28(5): 652-72. Doi: https://doi.org/10.1016/j.jvoice.2014.01.0 07.

17. Akinbode R, Lam KBH, Ayres JG, Sadhra S. Voice disorders in Nigerian primary school teachers. Occup Med 2014; 64(5): 382-386. Doi: https://doi.org/10.1093/occmed/kqu052.

18. Herrera Ariza JLA, Castro-Rojas JA. Disfonía ocupacional en docentes. Revisión de la literatura. Acta Otorrinolaringol Cir Cabeza Cuello [revista en la internet]. 2018; 46(1): 62$70 . \quad$ Disponible en: revista.acorl.org/index.php/acorl/article/downl $\mathrm{oad} / 89 / 70$.

19. Miri AK. Mechanical characterizacion of vocal fold tissue: review study. J Voice. 2014; 28(6): 657-67.

Doi: https://10.1016/j.jvoice.2014.03.001.

20. Pestana PM, Vaz-Freitas S, Manso MC. Prevalence of Voice Disorders in Singers: Systematic Review and Meta-Analysis. J Voice. 2017; 31(6): 722-727.

Doi: https://doi.org/10.1016/j.jvoice.2017.02.01 0 . 